\title{
Plasma Dexamethasone Levels in Children Given the Dexamethasone Suppression Test
}

\author{
Michael W. Naylor, John F. Greden, and Norman E. Alessi
}

To determine whether chis: whe demonstrate dexamethasone suppression test (DST) nonsuppression have lo ier plasma dexamethasone levels than DST suppressors, we administered the DST to 73 patients ranging in age from 5-14 years. Plasma dexamethasone levels and postdexamethasone cortisol levels were measured at 4:00 PM on day 2. We found: (1) DST nonsuppressors had significantly lower plasma dexamethasone levels $(p<0.03)$ than suppressors; similar trends were observed when the population was divided into depressed and nondepressed patients; (2) $\mathrm{mg} / \mathrm{m}^{2}$ dose of devimethasone was directly correlated with plasma dexamethasone $(\mathrm{p}<0.003)$ and inversely correlated with postdexamethasone plasma cortisol levels ( $\mathrm{p}<0.04$ ); and (3) a statistically significant inverse correlation between plasma dexamethasone leveis and postdexamethasone cortisol levels $(\mathrm{p}<0.04)$. Our findings show that plasma dexamethasone levels are important in evaluating DST results in psychiatrically disturbed children and suggest that dexamethasone dosage, $r$ use in the DST in children might be better calculated in terms of body surface area.

\section{Introduction}

Plasma dexamethasone levels are an important variable in understanding hypothalamicpituitary-adrenal (HPA) axis dysregulation in psychiatrically disturbed adults. Investigators have found that depressed adults who are nonsuppressors on the dexamethasone suppression test (DST) have significantly lower plasma dexamethasone concentrations than depressed patients and control subjects who are DST suppressors (Johnson et al. 1984; Arana et al. 1984; Maguire et al. 1987). Several explanations have been advanced for this observation. Lowy and Meltzer (1987) raised the possibility that bioavailability of dexamethasone plays a major mole in determining DST suppressor status and have suggested that simultaneous measurement of plasma dexamethasone and cortisol levels are needed to evaluate DST results. Alternatively, Holsboer and associates (1986a and b) concluded that the low plasma dexamethasone levels in nonsuppressing adult depressives were due to accelerated metabolism of dexamethasone rather than impaired bioavailability, and suggested that the abnormality of glucocorticoid necabolism in depressives with DST nonsuppression is state-dependent.

Although the plasma dexamethasone variable has not been studied in children, the

From the University of Michigan Department of Psychiatry, Child and Adolescent Psychiatric Hospital (M.W.N., N.E.A.) and the Michigan Depression Program (J.F.G.), University of Michigan, Ann Arbor, Michigan.

Address reprint requests to Dr. Michael W. Naylor, Department of Psychiatry, Box 0706, 1500 East Medical Center Drive, Ann Arbor, MI 48109-0706.

Received October 20, 1988; revised January 13, 1989. 
association between suppressor status and dexamethasone dose has been. In a study of 97 children aged 3 to 16 years, Doherty and co-workers (1986) found no significant difference in the percentage of depressed children who failed to suppress at high, medium, or low doses of dexamethasone based on body weight.

We undertook this study to examine the relationship between plasma dexamethasone levels and DST suppressor status in children. We hypothesized that: (1) psychiatrically disturbed children with DST nonsuppression would have lower plasma dexamethasone levels than DST suppressors regardless of diagnosis; and (2) dexamethasone dosage would ccrrelate directly with plasma dexamethasone levels and inversely with plasma cortisol levels.

\section{Methods}

\section{Patients}

Seventy-three children (aged 5-14 years) admitted to the Diagnostic and Research Unit at the University of Michigan Hospitals were administered the dexamethasone suppression test (DST), regardless of diagnosis. Exclusion criteria for the DST werr as described by Carroll et al. (1981). Demographic data are presented in Table 1. Major depressive disorder was the primary diagnosis in 14 children, dysthymic disorder in 11, adjustment disorder with depressed mood in 2, attention deficit clisorder with hyperactivity in 11 , anxiety disorder in 6, conduct disorder in 13, oppositional disorder in 3, schizophrenia in 1 , adjustment disorder with mixed disorder of mood and conduct in 3 , pervasive developmental disorder in 3, psychosomatic disorder in 3, and personality cisorder in 2.

We initially divided the population into DST suppressors and nonsuppressors and subsequently subdivided the population into depressed (major depressive disorder and dysthymic disorder) and nondepressed (all other diagnoses). The 2 children diagnosed as having an adjustment disorder with depressed mood were assigned to the nondepressed group (both were DST suppressors, and analysis of the data with these 2 children categorized as depressed did not change the results).

\section{Diagnostic Procedure}

Each child was rigorously evaluated medically and psychiatrically. Height and weight were recorded and body surface area was determined according to the method of Gehan and George (1970). Each patient and his or her family were interviewed by a senior staff ps, niatrist and a fellow in child psychiatry. Developmental and family history were obtained in the initial interview, and academic history was obtained from the child's school teaciler. Further diagnostic information obtained during the first 2 weeks of hospitalization included neuropsychological and psychological testing, milieu observations, and reports from the hospital's school. Consensual diagnoses were made after 2 weeks of hospitalization based on all available information according to unmodified DSM-III criteria.

\section{DST Procedure}

The DST was performed within the first week of hospitalization. All patients were medication free for at least 2 weeks before having the DST. After we obtained informed consent from the parents and assent from the child when possible, we drew a baseline 


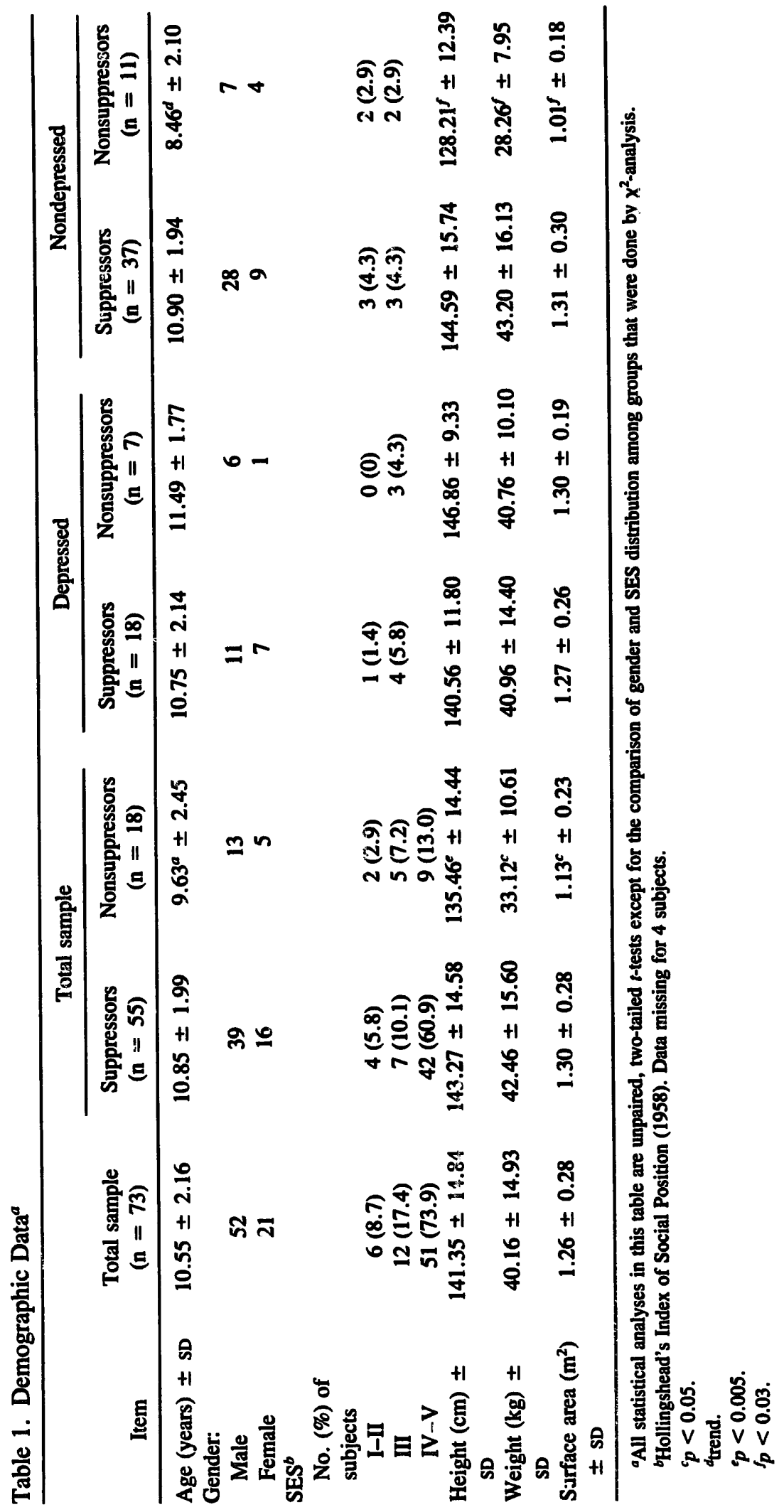


cortisol level at 11:00 PM on day 1, then gave each subject dexamethasone. For children weighing less than $36 \mathrm{~kg}$, we administered $0.5 \mathrm{mg}$ of dexamethasone as described by Poznanski et al. (1982). This dosage was based on the rationale that the children would receive approximately the same dose of dexamethasone on a $\mathrm{mg} / \mathrm{kg}$ basis that an averagesized adult would receive. Children weighing $36 \mathrm{~kg}$ or more were given $1.0 \mathrm{mg}$ as described by Carroll et al. (1981). Thirty-eight children received $0.5 \mathrm{mg}$ of dexamethasone whereas 35 received $1.0 \mathrm{mg}$. Children were observed closely by the nursing staff to assure they had taken the dexamethasone tablets. Plasma cortisol, obtained on day 2 at 4:00 PM, was measured by a competitive protein binding assay with interassay coefficients of variation of 7.6, 6.2, and $5.0 \%$ at $4.0,8.0$, and $16.0 \mu \mathrm{g} / \mathrm{dl}$ of cortisol, respectively, and intra-assay coefficients of variation of $7.1,5.8$, and $4.7 \%$ for the same plasma cortisol values. DST nonsuppression was defined as a postdexamethasone plasma cortisol $\geqslant 5.0$ $\mu \mathrm{g} / \mathrm{dl}$ at 4:00 PM. Concurrent plasma dexamethasone levels were obtained and quantified by a radioimmunoassay technique using rabbit antiserum produced against a bovine serum. albumin conjugate of dexamethasone hemisuccinate. The rabbit antiserum for the DST assay was provided by Merck Sharpe and Dohme. The sensitivity of the assay is $100 \mathrm{pg} /$ $\mu l$ using $100 \mu l$ of plasma and is reliable at plasma dexamethasone levels greater than or equal to $10 \mathrm{ng} / \mathrm{dl}$. The assay is highly specific for dexamethasone with no cortisol cross-reactivity until the concentration of cortisol exceeds $16-18 \mu \mathrm{g} / \mathrm{dl}$. Interassay coefficients of variation are 6.5 and $5.0 \%$ at 109 and $380 \mathrm{ng} / \mathrm{dl}$ of dexamethasone, respectively. The intra-assay coefficients of variation are 5.5 and $4.8 \%$ for the same values.

\section{Data Analysis}

To test the hypothesis that psychiatrically hospitalized children with DST nonsuppression have lower plasma dexamethasone levels than suppressors, we divided the total sample into DST suppressors and nonsuppressors and compared plasma dexamethasone levels between them using an unpaired, one-tailed $t$-test. We chose a one-tailed test as we postulated a specific directionality to the results and to reduce the likelihood of a Type I error, (incorrectly rejecting a true hypothesis). To determine whether the diagnosis of depression was a significant variable, we further subdivided the sample into depressed and nondepressed subjects and conducted the same analyses.

To analyze the data continuously as well as dichotomously, and to test the hypothesis that dexamethasone dosage would correlate directly with plasma dexamethasone and inversely with postdexamethasone cortisol levels, we calculated Pearson's $r$ correlation coefficients using dexamethasone dosage as one variable and plasma dexamethasone wi plasma cortisoi, respectively, as the second variable. Because plasma dexamethasone levels and postdexamethasone cortisol levels were not normally distributed, we log transformed the data before all analyses. For all statistical analyses, we accepted $p$ values of $<0.05$ as significant.

\section{Results}

\section{Plasma Dexamethasone Levels}

DST nonsuppressors had significantly lower $(p<0.03)$ plasma dexamethasone levels than suppressors (Table 2). When the total sample was divided according to the presence or absence of depression, both depressed and nondepressed DST nonsuppressors 


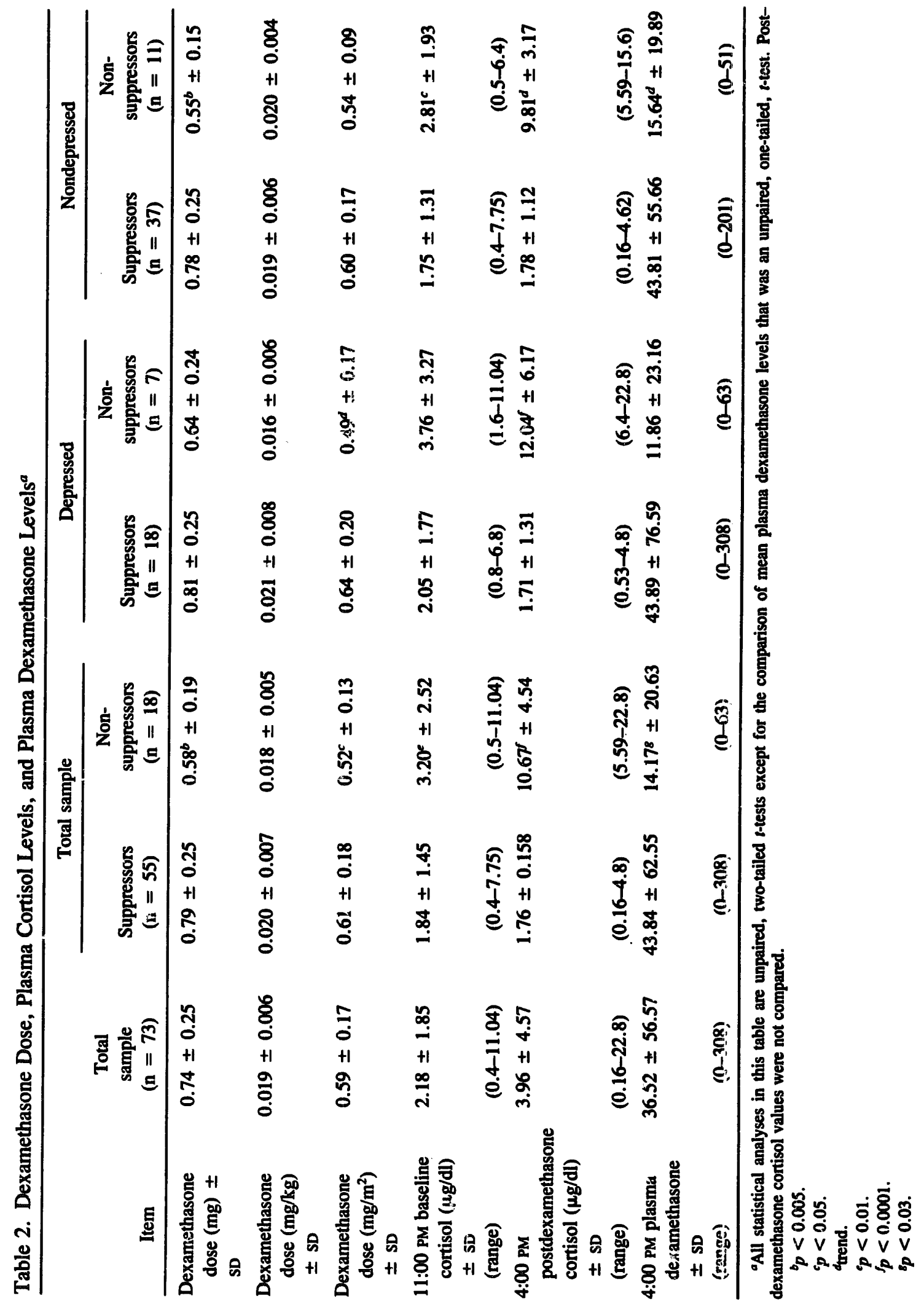




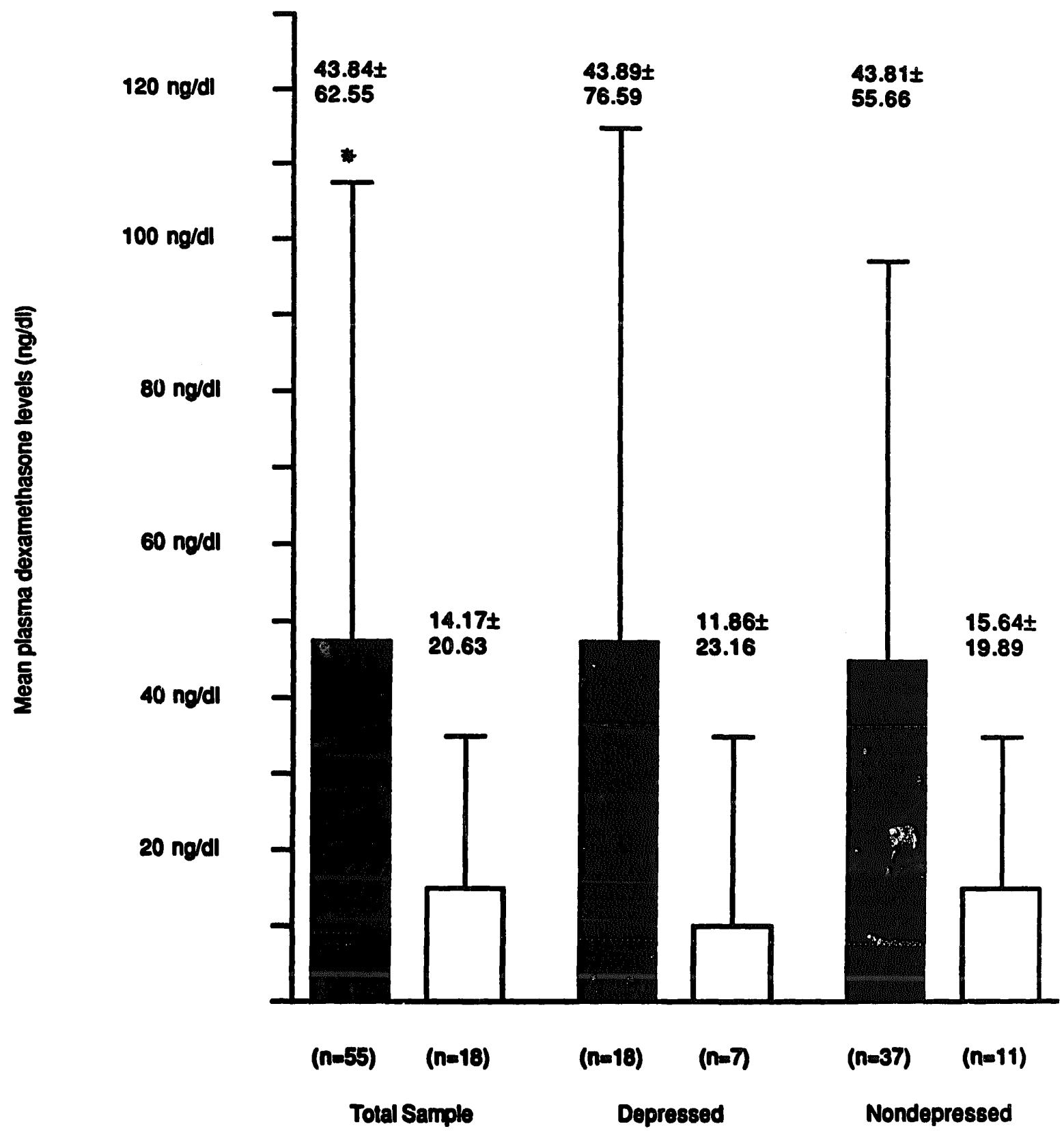

Figure 1. Mean plasma dexamethasone levels in suppressors ( $(\square)$ and nonsuppressors (ㅁ). *Unpaired, onetailed, $t$-test; $p<0.03$.

showed a similar trend towards having lower plasma dexamethasone levels; however, these findings failed to reach statistical significance (Figure 1). Fifteen of the 18 nonsuppressors received $0.5 \mathrm{mg}$ of dexamethasone (5 with depression and 10 with other diagnoses). Of the 3 nonsuppressors given $1.0 \mathrm{mg}, 2$ were depressed. $\chi^{2}$-analysis of the data revealed no differences in gender distribution between the various groups, suggesting that gender was not a major variable affecting plasma dexamethasone levels or DST suppressor status. 
Table 3. Correlation Among Dexamethasone Dose, Plasma Dexamethasone Levels, and Postdexamethasone Plasma Cortisol Levels

\begin{tabular}{|c|c|c|c|c|c|c|}
\hline & \multicolumn{2}{|c|}{$\begin{array}{c}\text { Total sample } \\
(\mathrm{n}=73)\end{array}$} & \multicolumn{2}{|c|}{$\begin{array}{l}\text { Nonsuppressors } \\
(\mathbf{n}=18)\end{array}$} & \multicolumn{2}{|c|}{$\begin{array}{l}\text { Suppressors } \\
(\mathrm{n}=55)\end{array}$} \\
\hline & $\begin{array}{c}\text { Plasma } \\
\text { dexamethasone }\end{array}$ & $\begin{array}{l}\text { Plasma } \\
\text { cortisol }\end{array}$ & $\begin{array}{c}\text { Plasma } \\
\text { dexamethasone }\end{array}$ & $\begin{array}{l}\text { Plasma } \\
\text { cortisol }\end{array}$ & $\begin{array}{c}\text { Plasma } \\
\text { dexamethasone }\end{array}$ & $\begin{array}{l}\text { Plasma } \\
\text { cortiso }\end{array}$ \\
\hline $\begin{array}{r}\text { Dexamethasone } \\
\text { dose }(\mathrm{mg} / \mathrm{kg})\end{array}$ & +0.09 & -0.04 & +0.18 & +0.31 & -0.04 & +0.02 \\
\hline $\begin{array}{r}\text { Dexamethasone } \\
\text { dose }\left(\mathrm{mg} / \mathrm{m}^{2}\right)\end{array}$ & $+0.35^{a}$ & $-0.25^{b}$ & +0.40 & +0.40 & $+0.29^{b}$ & -0.19 \\
\hline Plasma cortisol & $-0.25^{b}$ & - & +0.24 & - & $-0.58^{c}$ & - \\
\hline
\end{tabular}

${ }^{a} p<0.003$.

$b_{p}<0.04$.

${ }^{c} p<0.0001$.

\section{Association Between Dexamethasone Dose, Plasma Dexamethasone, and Postdexamethasone Plasma Cortisol Levels}

As shown in Table $3, \mathrm{mg} / \mathrm{m}^{2}$ dose of dexamethasone was directly correlated with plasma dexamethasone levels for the total sample $(p<0.003)$ and for DST suppressors $(p<$ 0.04), and inversely correlated with 4:00 PM postdexamethasone plasma cortisol levels for the total sample $(p<0.04)$. There was no correlation between $\mathrm{mg} / \mathrm{kg}$ dexamethasone dose and plasrna dexamethasone concentrations or postdexamethasone cortisol levels. We found a statistically significant inverse correlation between plasma dexamethasone levels and postdexamethasone cortisol levels for the total sample $(p<0.04)$ and for DST supprezsors $(p<0.0001)$. We found no significant correlations between dexamethasone dosage and plasma dexamethasone or cortisol concentrations, or between plasma dexamethasone concentrations and postdexamethasone plasma cortisol levels in DST nonsuppressors.

As shown in Tables 1 and 2, DST nonsuppressors were significantly younger, lighter, and smaller, and received less dexamethasone, either absolute or expressed in terms of $\mathrm{mg} / \mathrm{m}^{2}$, than suppressors.

\section{Discussion}

The results of thr. study support our hypothesis that DST nonsuppression in children is associated with low plasma dexamethasone levels when compared with DST suppressors. Our hypothesis that dexamethasone dosage is directly correlated with plasma dexamethasone levels and inversely with plasma cortisol levels was also supported. $\mathbf{M g} / \mathrm{m}^{2}$, but not $\mathrm{mg} / \mathrm{kg}$, dexamethasone dose was directly correlated with plasma dexamethascne levels in the total sample and DST suppressors, and inversely correlated with postdexamethasone cortisol concentrations in the total sample. We demonstrated an inverse correlation between plasma dexamethasone levels and postdexamethasone plasma cortisol concentrations for the total sample. Interestingly, however, we found no significant correlation among dexamethasone dosage and plasma dexamethasone or cortisol concentrations, or between plasma dexamethasone levels and cortisol concentrations in DST nonsuppressors.

It is not possible with this study design to address the question of why plasma dexamethasone levels were lower in DST nonsuppressors than in suppressors. Dexamethasone 
dosage likely accounts for part of the difference. As a group, nonsuppressors received significantly smaller $\mathrm{mg}$ and $\mathrm{mg} / \mathrm{m}^{2}$ dexamethasone doses than DST suppressors. Age, height, weight, and size differences may also account for part of the difference. Nonsuppressors were younger, smaller, and lighter than suppressors, and it may be that younger, smaller children metabolize dexamethasone more rapidly than older, larger ones. There is only minimal current support for this hypothesis; however, Richter et al. (1983) found that toddlers have lower plasma dexamethasone concentrations than adults after administration of equivalent doses of dexamethasone when corrected for weight-which offers tentative support. Finally, the lack of significant correlation between plasma dexamethasone levels and postdexamethasone cortisol levels in DST nonsuppressors suggests that hypothalamic-pituitary-adrenal axis dysregulation and altered glucocorticoid metibolism may account for the observed difference in plasma dexamethasone levels between suppressors and nonsuppressors.

The findings of this study must be interpreted with caution. There were no normal controls for comparison with our patient subgroups. Additionally, patients were not randomized to dosage groups nor were they administered both dosages of dexamethasone. Finally, severity and duration of the illness wera not accounted for in the study design. Future studies are necessary to determine the influence of severity and duration of illness, age, and size on glucocorticoid metabolism and consequently, dexamethasone suppressor status in depressed children. Nevertheless, these data are consistent with those obtained from adults.

This study has several implications for researchers in child psychiatry. The effect of dexamethasone dose, and subsequently plasma dexamethasone concentrations, on postdexamethasone plasma cortisol levels and DST suppressor status underscores the need for standardization of the DST protocol in children. At least 5 dexamethasone dosage strategies have been used by child psychiatric researchers including the moditied $0.5 \mathrm{mg}$ DST (Poznanski et al. 1982), the standard $1.0 \mathrm{mg}$ DST (Carroll et al. 1981), assignment of dexamethasone dosage based on a strict weight cut-off (Jensen and co-workers 1985), the use of a $20 \mu \mathrm{g} / \mathrm{kg}$ dose for all patients (Geller et al. 1983), and assignment of dexamethasone dose based on pubertal status (Emslie and co-workers 1987). This lack of standardization makes generalization among studies very difficult. The use of a $\mathrm{mg} /$ $\mathrm{m}^{2}$ dose of dexamethasone may be warranted on the basis of our findings and may aid in the standardization of the DST in children. Meanwhile, simultaneous measurement of plasma dexamethasone and postdexamethasone cortisol levels is indicated to determine the effects of various plasma dexamethasone levels on DST suppressor status.

The authors wish to thank M. Hariharan, Ph.D., for providing plasma dexamethasone assay levels; Cynthia Pomerleau, Ph.D., for her editorial assistance; and Nancy Genero, Ph. D., Director of the University of Michigan Department of Psychiatry Biometrics Program, for assistance with the statistical analysis.

\section{References}

Arana GW, Workman RJ, Baidessarini RJ (1984): Association between low plasma levels of dexamethasone and elevated levels of cortisol in psychiatric patients given dexamethasone. Am J Psychiatry 141:1619-1620.

Carroll BJ, Feinberg M, Greden JF, Tarika J, Albala AA, Haskett RF, James NM, Kronfol Z, Lohr N, Steiner M, deVigne JP, Young E (1981): A specific laboratory test for the diagnosis of melancholia: Standardization, validation, and clinical utility. Arch Gen Psychiatry 38:1522. 
Doherty MB, Madansky D, Kraft J, Carter-Ake LL, Rosenthal PA, Coughlin BF (1986): Cortisol dynamics and test performance of the dexamethasone suppression test in 97 psychiatrically hospitalized children aged 3-16. J Am Acad Child Psychiatry 25:400-408.

Emslie GJ, Weinberg WA, Rush AJ, Weissenburger J, Parkin-Feigenbaum L (1987): Depression and dexamethasone testing in children and adolescents. J Child Neurol 2:31-37.

Gehan EA, George SI. (1970): Estimation of body surface area from height and weight. Cancer Chemother Rep 54:225-235.

Geller B, Rogol A, Knitter EI (1983): Preliminary data on the dexamethasone suppression test in

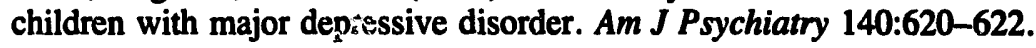

Hollingshead AB, Redlich FC (1958): Social Class and Mental Illness. New York: Wiley.

Holsboer F, Wiedemann K, Gerken A, Boll E (1986a): The plasma dexamethasone variable in depression: Test-retest studies and early biophase kinetics. Psychiatry Res 17:97-103.

Holsboer F, Wiedemann K, Boll E (1986b): Shortened dexamethasone half-life in depressed dexamethasone nonsuppressors (letter). Am J Psychiatry 43:813-815.

Jensen JB, Realmutto GM, Garfinkel BD (1985): The dexamethasone suppression test in infantile autism. J Am Acad Child Psychiatry 24:263-265.

Johnson GF, Huni G, Kerr K, Caterson I (1984): Dexamethasone suppression test (DST) and plasma dexamethasone levels in depressed patients. Psychiatry Res 13:305-313.

Lowy MT, Meltzer HY (1987): Dexamethasone bioavailability: Implications for DST research. Biol Psychiatry 22:373-385.

Maguire KP, Schweitzer I, Biddle N, Bridge S, Tiller JWG (1987): The dexamethasone suppression test: Importance of dexamethasone concentrations. Biol Psychiatry 22:957-967.

Poznanski EO, Carroll BJ, Banegas MC, Cook SC, Grossman JA (1982): The dexamethasone suppression test in prepubertal depressed children. Am J Psychiatry 139:321-324.

Richter O, Em B, Reinhardt D, Becker B (1983): Pharmacokinetics of dexamethasone in children. Ped Pharmacol 29:337. 